\section{O uso de contratos de gestão e incentivos profissionais no setor público de saúde}

\author{
The use of management contracts and professional \\ incentives in the public health sector
}

\begin{abstract}
Results-based management is a cornerstone of reform in public administration, including the health field, and has become the basis for other innovations such as the institutionalization of management contracts and the use of professional incentives. This review article aims to introduce and discuss the use of such management contracts in the public health sector. Management by results has developed means and tools that highlight the importance of shared responsibility and mutual commitment between workers and management-level directors. Thus, preset goals are negotiated among all the stakeholders and are evaluated periodically in order to grant professional incentives. It is necessary to improve the mechanisms for control and observation, to more precisely determine the healthcare and management indicators and their patterns, to train stakeholders in designing the plan, and to improve the use of professional incentives in order to effectively increase accountability vis-à-vis the desired results.
\end{abstract}

Health Services Administration; Health Management; Public Administration
Rafael Gomes Ditterich 1

Simone Tetu Moysés 2

Samuel Jorge Moysés 2

\section{Introdução}

Atualmente, diversos gestores em saúde têm enfatizado o papel da avaliação de desempenho nas ações e programas de saúde, com o objetivo de garantir a qualidade da atenção e subsidiar decisões que atendam às reais necessidades da população, alcançando resultados na produção de serviços e impacto na saúde 1 . A avaliação da situação de saúde, do trabalho em saúde e dos resultados das ações, decorre em grande parte das atividades de acompanhamento, monitoramento e avaliação realizadas com base nas informações produzidas no cotidiano da atenção 2 .

Tais atividades, embora sejam insuficientes para apreender a complexidade da produção social em saúde e as respectivas mudanças desejáveis, são essenciais para orientação dos processos de implantação, consolidação e reformulação das práticas de saúde 2 . O monitoramento do cotidiano deve ser constitutivo de uma gestão ampliada e responsável, no qual se faz necessário que o gestor exerça liderança democrática, trabalhando com sua equipe de maneira a compor os conhecimentos e habilidades do grupo na busca de um ambiente onde ocorra a sinergia e o desenvolvimento contínuo das pessoas.

Para melhor compreender o processo de liderança democrática é importante diferenciála dos outros tipos. Weber 3 diferenciou em três tipologias o comportamento do líder: liderança 
autoritária, liberal e democrática. A liderança autocrática possui sua ênfase centrada no líder, sendo ele quem fixa as diretrizes a serem seguidas e determina as técnicas a serem utilizadas, sem qualquer participação do grupo. Já a liderança democrática coloca sua ênfase tanto no líder como nos subordinados, sendo as diretrizes debatidas e as técnicas e processos escolhidos pelo grupo, assistidos pelo líder. Por sua vez, a liderança liberal coloca todo seu foco nos subordinados, e há liberdade completa, por parte dos colaboradores, para tomada de decisões.

Nessa perspectiva, o pressuposto é que o gestor de saúde, seja qual for sua esfera de atuação, deve ter uma visão ampliada de saúde, ser capaz de uma priorização adequada dos problemas e necessidades da comunidade, e de executar o modelo de atenção integral e da gestão participativa 4 .

Entretanto, as diversas realidades municipais colocam os gestores em saúde frente a vários desafios na busca de garantia da atenção universal, equitativa e integral, como: financiamento aquém do necessário, ausência de redes integradas de serviços, problemas na pactuação dos serviços contratados e conveniados, baixa sustentabilidade política e institucional, problemas na fixação de pessoal etc. Dessa forma há a necessidade de se fazer avançarem as lógicas que orientam os pactos entre os gestores dos serviços de saúde, criticando racionalidades produtivistas e resgatando uma matriz de valores em que o eixo principal seja o compromisso, a responsabilidade sanitária, solidária e compartilhada 5 .

Tais gestores têm a responsabilidade de articular o planejamento, negociar a execução, buscar o provimento de recursos, coordenar processos de trabalho e avaliar os resultados alcançados no território-população sob sua responsabilidade político-gerencial 6 . É, sem dúvida, uma tarefa demandante e desafiadora, sobretudo quando a gestão estratégica traz inexoravelmente como corolário o esforço de interpretar e se relacionar com as necessidades, angústias e representações das pessoas que habitam determinado território, e obter comprometimento e motivação das equipes de trabalho.

Tal conjunto complexo de atribuições e tarefas gera problemas de toda ordem, mas que podem se beneficiar da contribuição emanada da pesquisa, bem como de aportes tecnológicos capazes de oferecer soluções plausíveis. Se a gestão local em saúde, no Sistema Único de Saúde (SUS), tem a incumbência de agregar conhecimentos e tecnologias de gestão, com o objetivo maior de resolver problemas de saúde como função de equidade social, da eficiência e eficácia operativa e da democratização do acesso a ações e serviços de saúde, então há um fértil solo epistemológico a ser trabalhado.

Dentre os possíveis mecanismos de gestão em saúde e distintas realidades locais, algumas cidades brasileiras e serviços de saúde iniciaram um processo de revisão dos planejamentos locais e dos protocolos assistenciais, que culminaram com a implantação dos Contratos de Gestão e incentivos profissionais na gestão por resultados no SUS. O objetivo deste artigo é apresentar e discutir a utilidade e aplicabilidade de contratos de gestão e incentivos profissionais na gestão por resultados no setor público de saúde.

Para atingir tal objetivo, foi realizada uma revisão bibliográfica de artigos e relatórios de organismos internacionais (World Bank, World Health Organization, Organisation de Coopération et de Développement Économiques e European Observatory on Health Systems and Policies) sobre o uso de arranjos contratuais e incentivos profissionais nos sistemas e serviços de saúde. Foram consultadas as bases de dados LILACS, MEDLINE, SciELO, utilizando-se como palavras-chave de busca os termos: contracting, contracting management, contracting health systems, payment for performance, contratualização, contratos, gestão por resultados, contrato e desempenho, incentivos profissionais, incentivos financeiros e contrato de gestão.

\section{Os contratos de gestão nos serviços públicos de saúde}

O tema dos contratos de gestão em saúde é parte de uma importante agenda de discussão nos cenários brasileiro e internacional na organização de serviços de saúde 7,8,9,10,11. A contratualização de resultados é apontada como uma das principais estratégias da Nova Gestão Pública, ou seja, dentre as que mais promoveram mudanças substantivas na qualidade dos serviços públicos 12 .

A Nova Gestão Pública surge em um cenário de críticas ao modelo burocrático da administração pública e de novas exigências econômicas, políticas e sociais colocadas para o Estado 13,14. Tendo como objetivos flexibilizar a gestão e aumentar a prestação de contas da administração pública pela contratualização de resultados, propunha a instituição de mecanismos de pactuação de metas, indicadores e instrumentos de acompanhamento e de cobrança claramente definidos possibilitando maior controle por parte do Estado e da sociedade 14. Dessa forma, a gestão por resultados tem sido destacada como abordagem tipicamente marcada pela busca por 
eficácia, efetividade e eficiência na gestão dos serviços e na construção de políticas públicas.

Nessa lógica, os contratos de gestão surgem como instrumentos de implantação de uma administração por objetivos e busca inexorável por resultados no setor público, visando à qualidade de serviços e à eficiência das organizações. Esses contratos são compromissos gerenciais entre o governo e a diretoria de uma empresa ou setor estatal, com objetivos e metas periódicas, cuja supervisão é realizada pelo Estado 15,16.

Segundo André 17, o contrato de gestão é um instrumento que tem o propósito de induzir mudanças culturais que façam com que o Estado e cada uma das entidades de interesse social dele dependente ou a ele vinculada construam e mantenham níveis elevados de: (a) efetividade: atingindo os resultados sociais aspirados pela sociedade, inclusive oferecendo-lhe serviços de interesse social compatíveis com suas necessidades em extensão, qualidade e preços; (b) eficácia: comprometendo-se política e institucionalmente com um competente planejamento e com o cumprimento responsável da sua execução; (c) eficiência: usando com economia, zelo e dedicação os bens e os recursos públicos.

A contratualização de resultados tem sido também considerada como uma nova forma administrativa de controle interno na gestão pública e mobilizadora de processo contínuo de aperfeiçoamento e de mudança gerencial 18 . A principal questão do modelo de contratualização é a definição de resultados por meio de indicadores e metas, no qual implica um processo permanente de avaliação e ajustes. Não se trata, portanto, de um contrato estanque, mas sim de uma pactuação, seguida de apresentação de resultados, análise e, se necessário, de repactuação 19 .

O contrato de gestão nos serviços de saúde tem como finalidades: estimular a descentralização da gestão; dar mais responsabilidade aos gestores e equipes locais; controlar os desempenhos quantitativo e qualitativo dos prestadores; ajudar no planejamento local, estimular a utilização de ferramentas e tecnologias de informação; melhorar a gestão da clínica, gestão da patologia e a gestão do caso; focalizar as necessidades em saúde da população do território sob sua responsabilidade (no caso da atenção primária); buscar o envolvimento e compromisso de toda a equipe na busca de resultados, estimular a cultura de negociação; proporcionar mais transparência junto ao controle social 20,21.

Por conseguinte, a contratualização dos serviços de saúde deve ter como objetivo uma melhor resposta às necessidades e expectativas de saúde da população, por meio de uma prestação de ser- viços eficiente e de qualidade, com um processo bem definido 11. O processo de contratualização pressupõe a definição de demanda e objetivos, metas quantitativas e qualitativas por serviços, obrigações e responsabilidades para cada parte envolvida, critérios e instrumentos de monitoramento, avaliação de resultados e cumprimento de metas estabelecidas, mecanismos de participação e controle social, além da regulação do SUS como um todo 22.

Para a efetivação desse processo, Araújo 10 comenta que o contrato de gestão tem de apresentar: metas, meios, controle e incentivos. Metas bem definidas servem para orientar a ação de gestão; os meios criam condições para o alcance de metas; o controle permite acompanhar os processos, a alocação de recursos, avaliar a implantação das ações; e os incentivos constituem os elementos mobilizadores do comportamento humano em direção das metas.

Os contratos de gestão devem ser negociados e acordados entre as partes, como dispositivos de diálogo e contratação de compromissos e responsabilidades em torno de objetivos e metas afinadas com as necessidades entre os gestores e trabalhadores da saúde. É importante que haja interlocução entre os sujeitos envolvidos para construir mudanças, gerando novos padrões de relação e comunicação no âmbito das organizações/serviços de saúde ${ }^{23}$. De acordo com Robles \& Fischmann 24 , o processo de implantação dos Contratos de Gestão constitui-se numa forma de aprendizado mútuo do governo com os contratados, de modo a se adequar práticas e procedimentos e exercitar-se condições de negociação e o conhecimento de condicionantes existentes para ambas as partes.

A utilização de contratos de gestão tem sido comum na parceria estabelecida com o terceiro setor, como entre as Organizações Sociais (OS) e o Governo na gestão de vários hospitais, principalmente no Estado de São Paulo. Essa ferramenta, atualmente também vem sendo utilizada por alguns governos municipais e estaduais, no estabelecimento de pactuações dentro de suas secretarias e órgãos, como forma de maior participação e corresponsabilidade dos atores envolvidos na garantia da qualidade do cuidado e dos serviços de saúde.

Entretanto, nas duas condições citadas, o uso do contrato de gestão apresenta configurações diferenciadas. Os contratos endógenos são aqueles em que o "contratante" e o "contratado" são instituições/entidades estatais. Por exemplo, os contratos que secretarias municipais de saúde estabelecem com suas unidades de prestação de serviços - equipes de saúde da família, unidades ambulatoriais, hospitais, entre outros, ou com 
unidades administrativas descentralizadas (distritos sanitários) que permanecem, entretanto, como organismos estatais subordinados 25. Os contratos endógenos instituídos internamente nas instituições públicas brasileiras têm sido também denominados de Termo de Compromissos, cuja lógica operacional e fundamentos legais derivam, em grande parte, da experiência na definição de metas e resultados dos contratos de gestão utilizados nas OS 26. Por outro lado, os contratos exógenos são aqueles em que o ente contratante é uma instituição estatal que se relaciona com entes prestadores externos, como instituições públicas não estatais, instituições privadas filantrópicas ou instituições privadas lucrativas 27 .

Para o Banco Mundial 28, com sua crítica obstinada à quantidade e qualidade do gasto público em políticas sociais, os municípios devem aplicar mecanismos para fortalecer a responsabilização, tais como contratos de gestão que induzam os administradores a focarem em objetivos específicos e resultados mensuráveis. Esse instrumento, o contrato de gestão, poderia servir como mecanismo de base para o planejamento, monitoração e avaliação. Uma maior autonomia para unidades de saúde específicas deveria ser articulada com expectativas claras de desempenho (metas) e prestação de contas.

Contudo, uma estratégia fundamental para o processo de contratualização é estabelecer um método de acompanhamento avaliativo, cuidando para que as metas sejas aferidas (em seu cumprimento), não no sentido de "fiscalização de seu alcance absoluto", mas no que se refere como avaliação formativa capaz de ir incluindo as razões que explicam seu maior ou menor êxito, subsidiando regulações no processo de repactuação de metas 29 .

Lima 30 aponta que a utilização dos arranjos contratuais também envolve custos na sua implantação e manutenção. Há um custo de transação para preparar o contrato - o tempo dos atores utilizado para negociar, para levantar e analisar as informações necessárias à elaboração do contrato, para definir metas e indicadores; para monitorar e avaliar o contrato; e para bancar os incentivos presentes no contrato.

Costa-e-Silva et al. 31 argumentam que os municípios devem apostar em um modelo de gestão negociado, de ajustamento mútuo e comunicativo, e de um conjunto de práticas que intensifiquem o espírito de gestão por compromissos. Para que o processo de gestão orientado por resultados ocorra, os governantes devem criar mecanismos de aferição do desempenho, da satisfação dos usuários e de controle social, com incentivos profissionais vinculados à pro- dutividade e eficiência que, articulados, configuram as condições indispensáveis para a responsabilização dos gestores públicos 10 .

\section{A utilização de incentivos profissionais na produção do cuidado e como ferramenta gerencial nos serviços de saúde}

A utilização de incentivos profissionais adquire grande interesse nessa nova abordagem na gestão por resultados e de recursos humanos, tendo em vista os desafios apresentados atualmente pelas reformas do setor de saúde em curso no mundo 32,33 .

A proposta de sistemas de incentivos tem sido definida como o componente da estratégia gerencial constituído pelo conjunto de estímulos, financeiros ou não, que visam a ajustar e otimizar os componentes do processo produtivo nos serviços de saúde, que é extremamente complexo e deve ter como objetivo principal os interesses e necessidades dos usuários 34 .

Alguns autores defendem que os incentivos podem ajudar no alcance dos objetivos da política de saúde, favorecendo o acesso aos serviços de saúde necessários aos cidadãos, garantindo alta qualidade do atendimento e melhoria da equidade, bem como promovendo a utilização efetiva e eficiente dos recursos de saúde 35,36 . No entanto, embora programas de incentivos estejam sendo utilizados no planejamento e implementação de ações e serviços de saúde, poucas são as evidências existentes sobre a sua efetividade ou as suas potenciais consequências intencionais e não intencionais (externalidades), com os respectivos efeitos sobre a relação profissional/paciente e impactos sobre a saúde da população 33,37,38.

Entretanto, o fato é que formuladores de políticas, planejadores e gestores continuam buscando meios efetivos para recrutar e reter pessoal na área da saúde. A Organização Mundial da Saúde 39 calculou uma escassez global de 4,3 milhões de trabalhadores de saúde em todo o mundo. Traduzindo isso em termos de acesso aos cuidados em saúde, tal escassez pode significar que mais de um bilhão de pessoas não têm nenhum acesso a cuidados profissionais. Alguns acreditam que um modo para alcançar melhor equacionamento gerencial-trabalhista, nessa questão, seria desenvolver e implementar esquemas de gestão de pessoal com incentivos profissionais 40 .

No caso brasileiro, especialmente na atenção primária e na Estratégia Saúde da Família, muitos municípios sofrem a dificuldade de incor- 
porar e reter profissionais de saúde, e as razões são diversas. Estudo realizado com dirigentes de municípios com mais de 100 mil habitantes 41 , aponta que $60 \%$ das cidades avaliadas apresentam alguma forma de utilização de incentivos como forma de retribuição direta ou indireta para os trabalhadores de saúde. Atualmente, existe a necessidade de que os planos de cargos, carreiras e salários dos profissionais de saúde contemplem tipos de vínculos empregatícios e formas de remuneração compatíveis com as funções típicas de carreira de saúde e promovam, entre outras, a fixação do trabalhador (incluindo uma política de incentivos) e a possibilidade de vínculos flexíveis e adaptáveis a condições locais, pactuados entre trabalhadores, gestores e sociedade. Entretanto, atualmente os incentivos profissionais também têm sido aplicados para garantir a melhoria da qualidade do cuidado em saúde.

Para que um o programa de incentivos seja eficaz para os trabalhadores da saúde, ele deve apresentar as seguintes características: (a) tem de ter objetivos claros; (b) ser realista e aplicável; (c) refletir as necessidades e as preferências dos profissionais de saúde; (d) ser bem desenhado estrategicamente e apto para os seus fins; (e) estar adequado ao contexto local; (f) ser justo, equitativo e transparente; (g) pode ser medido e acompanhado; (h) incorporar elementos financeiros e não financeiros 40 .

Chiavenatto 42 acredita que a valorização do trabalhador pode ser realizada por diferentes meios capazes de recompensar o trabalho realizado: recompensas financeiras diretas, como salário, prêmios, comissões; recompensas financeiras indiretas em forma de férias, gratificações, horas-extras, 13o salário, adicionais; recompensas não financeiras oferecidas em forma de oportunidades de desenvolvimento, reconhecimento, segurança no emprego, qualidade de vida no trabalho, promoções, liberdade e autonomia.

A operacionalização de incentivos profissionais financeiros implica os seguintes princípios básicos 34: (a) é um mecanismo de gratificação do trabalho que se estabelece a partir de uma remuneração básica, não sendo, portanto, substitutivo do salário; (b) sua aplicação só deve ocorrer quando metas e indicadores foram alcançados, não podendo transformar-se numa gratificação permanente ou automática.

Os incentivos são ganhos extras que visam a mobilizar os trabalhadores para o alcance de determinadas metas ou reforço de determinados comportamentos, não devem ser adotados como mecanismos de equiparação salarial entre diferentes categorias profissionais ou compensação de defasagens na remuneração dos trabalhadores da saúde 32 .
De acordo com Cecílio et al. ${ }^{43}$, ao se adotar uma política de avaliação de desempenho que resulte em pagamento de gratificação financeira para os trabalhadores da saúde, está sendo proposta uma cadeia de apostas, na qual ao se cumprir tal conduta espera-se que ocorra um determinado resultado que pode desencadear um novo processo ou produto em saúde. No entanto, a utilização de sistemas de incentivos não deve jamais ser confundida com a lógica de mercado na produção de serviços de saúde, nem, tampouco, assemelhar-se com a remuneração por unidades ou atos praticados. A direcionalidade desses sistemas deve sim apontar para a satisfação dos usuários e ao atendimento de necessidades de saúde, o que inclui as dimensões de cobertura e impacto dos serviços prestados 34 .

Também há que se destacar que o simples fato de premiar ou recompensar os trabalhadores da saúde com base em resultados, não vai garantir automaticamente maior e melhor desempenho na realização do trabalho, bem como provocar mudanças duradouras nas atitudes e comportamentos nos cuidados em saúde 32 . Por isso, a utilização por longo período do incentivo profissional, pode provocar o risco da universalização da premiação, que pode induzir um comportamento oportunista e conservador na definição de metas, contribuindo negativamente para sua efetividade e transformando o prêmio num simples 14o salário 44 . Santana 34 ainda lembra a importância da gestão definir a forma e os meios de implantar, revisar e atualizar os critérios e os objetivos a serem alcançados com o sistema de incentivos e, também deve refletir as características do processo do cuidado nos serviços de saúde, marcadas por relações de trabalho complexas e diversificadas.

Dessa forma, é importante destacar que a presença dos trabalhadores da saúde junto com os dirigentes na definição de metas e objetivos se faz necessário para a garantia do compromisso mútuo e espírito de corresponsabilidade 45 .

De acordo com Cecílio et al. ${ }^{43}$, a gestão somente conseguirá intervir nos processos de trabalho, dentro dos serviços de saúde, quando os trabalhadores de saúde experimentarem um protagonismo ativo na condução da política de avaliação de desempenho. Desse modo, uma nova forma de gestão participativa vem sendo repensada, baseada em Unidades Funcionais (UF). Nessa abordagem, são agregados os serviços e setores que apresentavam afinidade de processos e produtos na gestão do cuidado. Com as UF se aposta na implantação de estrutura de poder descentralizado, com a profissionalização do quadro de pessoal para o exercício de funções estratégicas de coordenação e estabelecimento 
negociado de compromissos e metas com avaliação de desempenho 46.

Entretanto, outros autores comentam que a avaliação de desempenho, dependendo de como for avaliada, pode provocar um efeito negativo no processo de trabalho estabelecido (contraprodutividade), pois os profissionais de saúde poderão simplesmente blefar com as medidas e metas estabelecidas, ou ainda apostar em falhas no sistema de monitoramento/avaliação, de modo a continuarem recebendo os incentivos financeiros, em vez de se concentrarem na qualidade do atendimento ofertado aos usuários 9,11,37,47.

Como apontado, os atores envolvidos devem utilizar indicadores que meçam a qualidade e fomentem a discussão permanentemente, no cotidiano dos serviços de saúde, sendo que o fundamental é que as equipes de saúde ponham o tema da qualidade do atendimento como parte de suas preocupações e consigam avaliar aspectos substantivos do seu trabalho 43 . Para isso, segundo Santos et al. 45, os indicadores propostos na avaliação por desempenho não devem somente envolver aspectos quantitativos, mas pressupõem um determinado nível pactuado de qualidade no atendimento prestado.

Vale destacar que o processo de avaliação de desempenho não deve resumir-se somente a critérios exclusivos de premiação dos servidores, mas privilegiar o compromisso das equipes com os objetivos institucionais e vincular a gratificação ao alcance de metas de trabalho planejadas e pactuadas, que tenham como finalidade a garantia da eficiência do serviço de saúde e a qualidade do atendimento aos usuários 48 .

Por isso, nenhum sistema de avaliação de desempenho será eficaz, seja qual for o propósito, a não ser que as pessoas que estão sendo avaliadas saibam o que se espera delas e conheçam os critérios pelos quais estão sendo julgadas 38 .

De forma a direcionar os programas de pagamento por desempenho realizados no setor público, a Organização de Cooperação e Desenvolvimento Econômico (OCDE) 49 apontou as lições e os aprendizados das experiências internacionais em curso nos países europeus, que deveriam ser (re)pensados na implantação ou manutenção dos incentivos profissionais:

- O design deve levar em consideração a cultura do país e da organização. O equilíbrio entre fatores individuais e coletivos na remuneração é peça fundamental na gestão por desempenho. Esquemas focados em equipes apresentam efeitos mais positivos do que os focados somente em aspectos individuais;

- A remuneração variável é parte de um sistema mais amplo. Transparência em todo o processo é um fator fundamental para garantir o sucesso do programa que depende mais da qualidade e da forma de mediação do processo de mensuração do desempenho do que puramente da distribuição da remuneração;

- Problemas de implementação devem ser antecipados, devendo ser preparados os servidores e as chefias;

- O programa deve estar vinculado a uma gestão de pessoas fundada na delegação. É mais fácil vincular objetivos individuais aos da organização se há certa autonomia para os gerentes atuarem; - Não deve superestimar o significado e o impacto do programa de avaliação por desempenho na motivação. A avaliação por desempenho tem papel secundário como ferramenta de gestão para aumentar a motivação. Critérios como a satisfação no trabalho, oportunidades de promoção ou a flexibilidade de organização do trabalho são muito mais eficazes na motivação da equipe e na remuneração ao desempenho;

- Os programas devem ser aplicados em ambientes onde as relações de trabalho sejam fundadas na confiança. No ambiente de trabalho, os processos formais e informais devem ser equilibrados, deve haver diálogo e troca de informações, e a negociação deve ser possível e estimulada, dando prioridade ao respeito mútuo e transparência;

- A avaliação por desempenho deve ser utilizada como incentivo e estímulo a uma transformação mais abrangente. Os incentivos financeiros devem ser utilizados principalmente como estímulo e ponte para a introdução de uma mudança ampla de direção e organização, ao invés de ser apenas uma ferramenta motivacional para o pessoal.

Aos avaliar os itens apresentados pela OCDE e reproduzi-los ao contexto brasileiro, a diversidade regional e heterogenicidade existentes nos mais de 5.560 municípios no nosso país, dificilmente poderia se pensar em uma única forma ou/e estratégia de incentivos profissionais para que se tenha sucesso em todo o território nacional, por isto é importante identificar as várias experiências em curso para que, baseado nesse mix de estratégias, cada localidade crie seus mecanismos de aferição dentro da política de gestão por resultados baseada em contrato de gestão e uso de incentivos profissionais.

Ainda em muitas organizações do setor público no Brasil, quanto existe a política de incentivos na grande maioria há o predomínio de uma visão avaliativa que privilegia somente a produtividade, muitas vezes no sentido mais injustificado que se reduz à contagem de procedimentos e controle contábil, produzindo assim ambiguidades e conflitos no ambiente de trabalho, impedindo que os trabalhadores distin- 
gam outros objetivos mais nobres do processo de cuidado à saúde 38 . Por isso, os sistemas de gratificações financeiras devem romper com a ênfase frequentemente baseada somente na melhoria da produtividade, independentemente de impacto qualitativo das intervenções nos indicadores sanitários ou epidemiológicos, ou na consideração de quem tais serviços sejam realmente necessários 9,50.

No contexto do cuidado no SUS, quanto à questão da gestão de pessoas e confiança nos serviços de saúde, é fundamental se pensar o papel da liderança nas equipes de trabalho. A liderança deve produzir mudança e movimento nas organizações por meio da criação de uma visão, desenvolvimento de estratégias, alinhamento das pessoas, bem como a responsabilidade de inspirar e motivar o grupo 51. Matus 52, ao estudar sobre o poder nas organizações de saúde, afirma que este é compartilhado por vários núcleos de profissionais, reforçando a necessidade de aperfeiçoamento constante na comunicação e na capacidade de negociação das lideranças. Também é fundamental que a confiança entre os atores sociais envolvidos seja repensada como pré-requisito essencial para maximizar a qualidade e os resultados. Quando a confiança predomina é possível estabelecer entre as partes distintas uma associação e compartilhar objetivos e projetos 30,53. Segundo Lima ${ }^{30}$, o modelo contratual funciona melhor quando a confiança prevalece entre as partes e quando os esforços são empreendidos para a cooperação e o sinergismo das capacidades e potencialidades entre as partes envolvidas nas relações.

Outro aspecto que não pode ser negligenciado nas experiências nacionais é que sistemas de incentivos financeiros valorizem não só o desempenho individual, mas principalmente o trabalho em equipe, uma vez que os incentivos, neste caso, não devem ser concedidos individualmente 25 . Sutton \& McLean 54 comentam que para o sucesso no uso de incentivos financeiros é necessário que a avaliação dos resultados se concentre na produção do cuidado em saúde de forma integral realizada pela equipe multidisciplinar, com base em evidências que orientem o trabalho para o alcance dos melhores indicadores de saúde na população atendida.

\section{Algumas experiências de gestão desenvolvidas nos serviços públicos de saúde no Brasil}

A implantação do SUS impôs aos gestores buscarem novas e mais apropriadas formas de gestão em saúde. As instâncias deliberativas/executivas passaram por um efetivo processo de descentralização e as decisões foram deslocadas para os níveis locais, mais próximos dos usuários dos serviços de saúde.

Dentre essas novas formas de gestão em saúde, a contratualização por resultados vem sendo amplamente discutida nesse processo, principalmente pelo uso de contratos de gestão e incentivos profissionais. Na literatura nacional há relato de uso dessas ferramentas em diversos espaços do setor público em saúde no SUS, tais como: hospitais, redes de atenção em saúde municipais e estaduais, e agências reguladoras.

Em relação aos contratos de gestão, André 17 comenta que o setor saúde apresenta exemplo claro de insucesso do uso do contrato de gestão exógeno como ferramenta gerencial, como foi o caso do Plano de Atendimento à Saúde (PAS), criado na década de 1990 pela Prefeitura Municipal de São Paulo, de altos custos e desrespeito à equidade e à universalidade no atendimento. Segundo Narvai \& São Pedro 55, a estratégia do PAS foi de transferir para a iniciativa privada a gestão dos serviços de saúde no município, no qual as OS eram remuneradas por usuários em potencial (número calculado com base na população de determinada localidade), independentemente se prestavam ou não os serviços à população, bem como também ignoravam a participação social (controle público) na gestão em saúde. Em 2000, após a constatação de diversas irregularidades como política pública de saúde, o PAS havia se transformado em símbolo de incompetência, descrédito e corrupção no setor saúde.

Por outro lado, a experiência do Estado de São Paulo com as OS que atuam na gestão hospitalar desde 1998, merece maior debate. Segundo Sano \& Abrúcio 14 , o sucesso das OS tem a ver basicamente com três fatores. Primeiro, a flexibilização administrativa favoreceu um melhor gerenciamento. Segundo, a adoção dos contratos de gestão aumentou a capacidade de planejar e executar a avaliação das metas. Por fim, o Estado de São Paulo criou um núcleo burocrático dentro da Secretaria Estadual de Saúde que foi capaz de regular, monitorar, avaliar e, eventualmente, mudar o rumo do processo de contratualização. Entretanto, Tibério et al. 56 comentam que pode ser questionada a maior flexibilidade administrativa no modelo de OS no Estado de São Paulo, tendo em vista que as limitações ao empenho do orçamento com recursos humanos associadas ao estabelecimento de metas e resultados no contrato de gestão permitem pouca flexibilidade administrativa. Contudo, apontam como benefício a maior flexibilidade em relação às compras, que de certa forma amplia a capacidade de 
resposta da organização às mudanças nas demandas em saúde.

Dentre os serviços de saúde, o uso de contrato de gestão também foi proposto aos hospitais públicos de ensino. Segundo Reis 57, em 2004 o Governo Federal implantou o Programa de Reestruturação dos Hospitais de Ensino, que compreendeu a contratualização destes estabelecimentos. Essa foi a forma encontrada de estratégia para o enfrentamento da crise do setor, ao estabelecer novos modos de financiamento, de gestão e de articulação desses hospitais com o sistema de saúde, mediante contrato de gestão com o gestor local do SUS. O autor apontou como avanço proporcionado pela contratualização a mudança no perfil de financiamento, resultando em equilíbrio econômico-financeiro e no enfrentamento do endividamento, embora com intensidades e reflexos distintos para os hospitais estudados. No entanto, diretrizes para o ensino, educação permanente, pesquisa e incorporação tecnológica, fundamentais para a produção do novo hospital de ensino, foram finalidades claramente esquecidas na implementação da política, que também não foi capaz de proporcionar mudanças consideráveis em relação à qualificação da gestão e da assistência 57 .

Outro relato do uso de contrato de gestão pode ser identificado nas agências reguladoras, como no caso da Agência Nacional de Vigilância Sanitária (ANVISA). Segundo Moreira \& Costa 58, a gestão por resultados mediante contrato de gestão na ANVISA foi desenhada como novo arranjo político-organizacional num componente do sistema público de saúde, comprometido, simultaneamente, com o papel regulador (associado ao atributo eficiência) e com a finalidade de "promover a proteção da saúde da população" (vinculada à redução de riscos, efetividade e impacto). Os autores constataram que a finalidade de "promover e proteger a saúde da população”, afirmada na missão institucional, não apresentou estratégias consistentes de implementação que priorizassem a efetividade das ações realizadas. Constatou-se também, que o contrato de gestão e suas repactuações carecem de maior legitimidade ante a sociedade, pois não foram submetidos ao Conselho Nacional de Saúde, tampouco possuem instrumentos normativos que versem sobre o controle social e a sistemática de acompanhamento e avaliação. Verificou-se que a primeira versão do plano de metas de 1999, continha macroações "ambiciosas”, tanto em número quanto em factibilidade. Com o passar do tempo, agregaram-se pautas técnicas, consideradas mais "no terreno do possível”, focalizando-se nos processos de trabalho e na construção de metas para dar respostas mais rápidas ao segmento produtivo e adquirir credibilidade e legitimação.

Já na contramão da terceirização e das parcerias público-privadas, alguns municípios e estados federativos brasileiros adotaram o contrato de gestão endógeno como ferramenta gerencial na gestão por resultados na atenção primária, em especial em Curitiba (Paraná) e no Estado de Minas Gerais, como forma de maior participação e corresponsabilidade dos atores envolvidos, na garantia da qualidade do cuidado e dos serviços de saúde.

Em Curitiba, desde 2002, na organização da atenção à saúde no município foram estabelecidos contratos de gestão localmente designados como Termo de Compromissos (TERCOM). Tais contratos são construídos com base em quatro variáveis principais: os objetivos do sistema de serviços de saúde contidos nos planos de saúde; as evidências e o custo efetividade das tecnologias de saúde; a disponibilidade e capacidade de ajustar a oferta de serviços de saúde; e as necessidades, demandas e expectativas dos cidadãos 59. O TERCOM tem como objetivo ser uma estratégia de gestão voltada para resultados que valorizam principalmente a saúde familiar, o cuidado especial com portadores de patologias crônicas, os determinantes e condicionantes dos riscos populacionais e o reforço à importância do autocuidado, hábitos saudáveis de vida e da promoção da saúde. É importante destacar que o cumprimento do TERCOM é composto por mecanismos de monitoramento, avaliação e um sistema de incentivos chamado Incentivo ao Desenvolvimento da Qualidade (IDQ) 59,60,61. Esse sistema implantado em Curitiba tem sido avaliado como inovador, vem provocando mudanças de postura entre os profissionais, que passam de passivos para ativos e, principalmente, orientados para a busca de resultados. Melhorou o desempenho quantitativo e qualitativo dos serviços e dos indicadores locais de saúde com a descentralização da gestão, o que proporciona mais responsabilidade aos gerentes locais 59,60. Entretanto, Ditterich 62 verificou que há ainda lacunas importantes na concreta efetivação da gestão pactuada, sendo necessário que os profissionais de saúde se apropriem de todas as fases e etapas de planejamento, elaboração, execução e avaliação das ações e programas pactuados no TERCOM.

Na experiência do Estado de Minas Gerais, os conceitos e as práticas da gestão pública por resultados estão articulados a um sistema de remuneração variável ainda inédito no setor público brasileiro, do ponto de vista de sua abrangência. Cada órgão do Poder Executivo estadual assina um contrato de gestão baseado em 
indicadores de desempenho, chamado acordo de resultados, caracterizado pelo pagamento de bônus anuais aos servidores públicos, segundo o desempenho (resultado atingido) avaliado por uma comissão 44 . O modelo é operacionalizado a partir da assinatura de um acordo de resultados entre cada dirigente de órgão e o governador, no qual a organização assume compromissos a serem entregues para a sociedade. Esse acordo estabelece indicadores e metas que a organização se compromete a alcançar. Em contrapartida, são concedidas autonomias gerenciais e a possibilidade de recebimento de prêmio por produtividade aos servidores públicos, em caso de resultados satisfatórios em relação às metas pactuadas 44 . As ações no âmbito da saúde se distribuem em várias Áreas de Resultados, mas concentram-se mais fortemente na área denominada Vida Saudável, que tem como objetivos estratégicos, cada qual com suas respectivas metas de curto, médio e longo prazos: (1) universalização do acesso à atenção primária à saúde; (2) redução da mortalidade infantil e materna; (3) ampliação da longevidade da população adulta; (4) aumento da eficiência alocativa do sistema de atenção à saúde; e (5) ampliação do acesso ao saneamento básico 63 .

Sobre a questão dos incentivos profissionais, na literatura existem algumas experiências sendo relatadas na área da gestão hospitalar. Cecílio et al. 43, ao avaliarem a institucionalização de incentivos profissionais no Hospital São João Batista (HSJB) em Volta Redonda (Rio de Janeiro), verificaram que o HSJB conseguiu formular e implementar uma inovadora política de avaliação institucional, de forma regular e com significativo reforço nos salários dos seus trabalhadores, bem como conseguiu construir com o tempo uma planilha com bastante sensibilidade para aspectos substantivos da vida do hospital, com indicadores produzidos de forma regular, valendo-se da informação de boa qualidade e, mais importante, com alto grau de credibilidade junto aos trabalhadores. Por outro lado, é necessário reconhecer que a planilha não conseguiu avaliar a qualidade da atenção prestada em todas as suas dimensões, necessitando ser combinada com outros indicadores incorporados, de forma regular, à gestão do hospital. A política de avaliação de desempenho se consolidou como um importante dispositivo de gestão e um aliado fundamental da direção na condução do hospital. Sem dúvida, a política de avaliação é um instrumento de controle em rede-descentralizado e com pretensões de legitimidade - mas, certamente, uma potente forma de controle por parte da direção da organização, para além de qualquer consideração sobre os méritos dos “objetivos organizacionais", sejam eles a busca pura e simples de maiores lucros no setor privado ou a construção de um hospital do SUS, comprometido com a defesa da vida.

Sobre outra experiência na área de gestão hospitalar, Fontenele 38 relatou a percepção dos trabalhadores sobre a avaliação de desempenho desenhada no Hospital Geral de Fortaleza (Ceará) e constatou que os sujeitos pesquisados afirmam que não recebem retorno das avaliações, gerando descrença e nenhuma legitimidade ao programa de avaliação. Os resultados das avaliações deveriam subsidiar medidas administrativas e políticas internas de recursos humanos. Também se evidenciou desconhecimento generalizado acerca da metodologia, critérios e objetivos estabelecidos para as avaliações. A pesquisa apontou que a aliança entre avaliações e concessão de incentivos e gratificações financeiras, como rege o programa de avaliação, tem sido comprometida pelos critérios estratificadores das categorias profissionais, bem como da falta de imparcialidade das chefias nas decisões, inibindo a realização de avaliações mais coerentes e verdadeiras por parte dos servidores, diante de possíveis punições e retaliações.

Em Curitiba, a política de incentivo financeiro estabelecida na gestão da atenção primária pode ser apontada como uma importante aliada na busca da motivação e comprometimento dos profissionais de saúde com a inovação do processo de trabalho e alcance de metas/resultados pactuados com os gestores, bem como uma adequada ferramenta na busca da qualidade dos serviços de saúde. Entretanto, existe a necessidade de a gestão municipal aprimorar o retorno aos profissionais de saúde sobre os processos de avaliação individual realizada pelas respectivas chefias, assim como a avaliação da comunidade atendida pelas equipes de saúde, de modo a tornar mais efetivo o trabalho realizado 62 .

Com a implantação de novos mecanismos gerenciais no setor saúde, tais como o uso de contrato de gestão e incentivos profissionais, espera-se que estes provoquem mudanças no processo de trabalho e gerência.

A gestão por resultados só será possível quando existir corresponsabilidade e compromisso mútuo entre os trabalhadores e o nível diretivo. Por isso, as metas preestabelecidas devem ser pactuadas entre todos os atores envolvidos e avaliadas de forma periódica para que os incentivos profissionais sejam garantidos. Para efetivamente aumentar a responsabilização sobre os resultados desejados, com base nas experiências analisadas, é preciso aprimorar os mecanismos de controle e monitoramento, definir de forma mais precisa indicadores e seus padrões no campo da assistência e da gestão, capacitar 
as partes envolvidas na elaboração do plano e no seu monitoramento com o uso de incentivos profissionais.

Também existe a necessidade de se repensar a gestão por resultados de uma forma em que o processo de trabalho seja analisado sob o ponto de vista coletivo, e não somente na singularidade da produção individual. Por isso, é importante que a pactuação de resultados e o uso de incentivos profissionais sejam um investimento humano permanente, respeitando parâmetros não somente quantitativos e de produtividade, mas observando centralmente que a produção do cuidado em saúde com qualidade exige a humanização do trabalho, a satisfação dos usuários e a geração de impacto socioepidemiológico.

\section{Resumo}

A gestão por resultados constitui um dos pilares da reforma na gestão pública, inclusive na área da saúde, tendo como principais inovações: a institucionalização de contratos de gestão e a utilização de incentivos profissionais. O objetivo deste artigo de revisão de literatura é apresentar e discutir a utilidade e aplicabilidade de contratos de gestão e incentivos profissionais na gestão por resultados no setor público de saúde. A gestão por resultados só será possível quando existir corresponsabilidade e compromisso mútuo entre os trabalhadores e o nível diretivo. Por isso, as metas preestabelecidas devem ser pactuadas entre todos os atores envolvidos e avaliadas de forma periódica para que os incentivos profissionais sejam garantidos. $\mathrm{Pa}$ ra efetivamente aumentar a responsabilização sobre os resultados desejados é preciso aprimorar os mecanismos de controle e monitoramento, definir de forma mais precisa indicadores e seus padrões no campo da assistência e da gestão, capacitar as partes envolvidas na elaboração do plano e aperfeiçoar o uso de incentivos profissionais.

Administração de Serviços de Saúde; Gestão em Saúde; Administração Pública

\section{Colaboradores}

R. G. Ditterich participou da elaboração e redação do artigo. S. T. Moysés participou da discussão e da revisão do artigo. S. J. Moysés participou da elaboração e da revisão final do artigo.

\section{Agradecimentos}

À Pró-Reitoria de Pesquisa, Pós-graduação e Inovação da Universidade Federal Fluminense (PROPPI-UFF) pelo auxílio stricto sensu (Doutorado). 


\section{Referências}

1. Szwarcwald CL, Mendonça MHL, Andrade CLT. Indicadores de atenção básica em quatro municípios do Estado do Rio de Janeiro, 2005: resultados de inquérito domiciliar de base populacional. Ciênc Saúde Coletiva 2006; 11:643-55.

2. Felisberto E. Monitoramento e avaliação na atenção básica: novos horizontes. Rev Bras Saúde Matern Infant 2004; 4:317-21.

3. Weber M. Economia y sociedad. México DF: Fondo de Cultura Económica; 1992.

4. André AM, Ciampone MHT. Desafios para a gestão de unidades básicas de saúde. Rev Adm Saúde 2007; 9:16-21.

5. Silva SF, Dobashi BF. Um novo pacto no SUS. Divulg Saúde Debate 2006; 34:9-17.

6. Morozowski-Filho N, Sutile VM, Thomaz M, Cubas RF. Gestão local e saúde bucal. In: Moysés SJ, Silveira-Filho AD, Ducci L, Simão MG, Gevaerd SP, organizadores. Os dizeres da boca em Curitiba: Boca Maldita, Boqueirão, bocas saudáveis. Rio de Janeiro: Centro Brasileiro de Estudos de Saúde; 2002. p. 97-107.

7. Josephson GW, Karcz A. The impact of physician economic incentives on admission rates of patients with ambulatory sensitive conditions: an analysis comparing two managed care structures and indemnity insurance. Am J Manag Care 1997; 3:49-56.

8. Teixeira L, Bugarin M, Dourado MC. Intermunicipal health care consortia in Brazil: strategic behavior, incentives and sustainability. Int J Health Plann Manage 2006; 21:275-96.

9. Loevinsohn B. Performance-based contracting for health services in developing countries: a toolkit. Washington DC: World Bank; 2008.

10. Araújo MAD. Responsabilização pelo controle de resultados no Sistema Único de Saúde no Brasil. Rev Panam Salud Pública 2010; 27:230-6.

11. Leite JDBL, Souza HF, Nascimento ECV. Contratualização na saúde: proposta de um contrato único para os programas no Estado de Minas Gerais. Rev Adm Saúde 2010; 12:15-22.

12. Jann W, Reichard C. Melhores práticas na modernização do Estado. Revista do Serviço Público 2002; 53:31-50.

13. Araújo M, Sanchez OA. A corrupção e os controles internos do estado. Lua Nova 2005; 65:137-73.

14. Sano H, Abrúcio FL. Promessas e resultados do Nova Gestão Pública no Brasil: o caso das Organizações Sociais de Saúde em São Paulo. Revista de Administração de Empresas 2008; 48:64-80.

15. André AM. Contrato de gestão: contradições e caminhos da administração pública. Revista de Administração Pública 1995; 35:6-13.

16. El-Warrak LO. A contratualização no âmbito da gestão pública da saúde: a experiência da área de planejamento 4 do Município do Rio de Janeiro [Dissertação de Mestrado]. Rio de Janeiro: Escola Nacional de Saúde Pública Sergio Arouca, Fundação Oswaldo Cruz; 2008.

17. André AM. A efetividade dos contratos de gestão na reforma do estado. Revista de Administração Pública 1999; 39:42-52.
18. Giacomini $\mathrm{CH}$, organizador. Gestão para resultados em Curitiba: a experiência de contratualização. Curitiba: Instituto Municipal de Administração Pública; 2009.

19. Ribeiro AKA, Schlichting M. Fases de desenvolvimento dos contratos de gestão na Prefeitura de Curitiba. In: Giacomini CH, organizador. Gestão para resultados em Curitiba: a experiência de contratualização. Curitiba: Instituto Municipal de Administração Pública; 2009. p. 93-8.

20. Marty IK. Primeiras experiências de contratos de gestão em Curitiba: Secretaria Municipal da Saúde In: Giacomini CH, organizador. Gestão para resultados em Curitiba: a experiência de contratualização. Curitiba: Instituto Municipal de Administração Pública; 2009. p. 43-56.

21. Vézina M, Denis JL, Turgen J. Contracting: an appropriate strategic management tool at the local level? Infolleter - The Evolution of Health-Systems Management and Evaluation Practices 2006; 3:2-7.

22. Conselho Nacional de Secretarias Municipais de Saúde. Reflexões aos novos gestores municipais de saúde. Brasília: Conselho Nacional de Secretarias Municipais de Saúde; 2009.

23. Santos-Filho SB, Figueiredo VON. Contratos internos de gestão no contexto da política de humanização: experimentando uma metodologia na referência de cogestão. Interface Comunic Saúde Educ 2009; 13 Suppl 1:615-26.

24. Robles LT, Fischmann AA. Contratos de gestão no Estado de São Paulo: o caso das empresas estatais não-financeiras. Revista de Administração Pública 1995; 35:52-64.

25. Costa-e-Silva V. O processo de implantação do Sistema Integrado de Serviços de Saúde em VitóriaES: contribuição à discussão da integralidade na atenção à saúde [Dissertação de Mestrado]. Rio de Janeiro: Escola Nacional de Saúde Pública Sergio Arouca, Fundação Oswaldo Cruz; 2004.

26. Barbosa NB, Elias PEM. As organizações sociais de saúde como forma de gestão público/privado. Ciênc Saúde Coletiva 2010; 15:2483-95.

27. Mendes EV. Os sistemas de serviços de saúde: o que os gestores deveriam saber sobre essas organizações complexas. Fortaleza: Escola de Saúde Pública do Ceará; 2002.

28. Banco Mundial. Governança no Sistema Único de Saúde (SUS) do Brasil: melhorando a qualidade do gasto público e gestão de recursos. Brasília: Banco Mundial; 2007.

29. Santos-Filho SB. Articulando planejamento e contratos de gestão na organização de serviços substitutivos de saúde mental: experiência do SUS em Belo Horizonte. Saúde Debate 2008; 32:172-81.

30. Lima SML. Possibilidades e limites da contratualização no aprimoramento da gestão e da assistência em hospitais: o caso dos Hospitais de Ensino no Sistema Único de Saúde brasileiro [Tese de Doutorado]. Rio de Janeiro: Escola Nacional de Saúde Pública Sergio Arouca, Fundação Oswaldo Cruz; 2009. 
31. Costa-e-Silva V, Rivera FJU, Hortale VA. Projeto Integrar: avaliação da implantação de serviços integrados de saúde no Município de Vitória, Espírito Santo, Brasil. Cad Saúde Pública 2007; 23:1405-14.

32. Fekete MC, Almeida MJ. Sistemas de incentivo ao trabalho em saúde. Espaç Saúde 2000; 2:1-21.

33. Glickman SW, Peterson ED. Innovative health reform models: pay-for-performance initiatives. Am J Manag Care 2009; 15:S300-5.

34. Santana JP. A gestão do trabalho nos estabelecimentos de saúde: elementos para uma proposta. In: Santana JP, Castro JL, organizadores. Capacitação em desenvolvimento de recursos humanos de saúde CADRHU. Natal: EDUFRN; 1999. p. 387-400.

35. Smith PC, York N. Quality incentives: the case of U.K. general practitioners. Health Aff 2004; 23:112-8.

36. Langenbrunner JC, Cashin C, O’Dougherty S. Designing and implementing health care provider payment systems: how-to manuals. Washington DC: World Bank, 2009.

37. Snyder L, Neubauer RL. Pay-for-performance principles that ensure promotion of patient-centered care: an ethics manifesto. Ann Intern Med 2007; 147:792-4.

38. Fontenele MFM. Gestão do desempenho humano: um estudo de caso em um Hospital Geral de Fortaleza (CE). Ciênc Saúde Coletiva 2010; 15 Suppl 1:1315-24.

39. World Health Organization. The world health report 2006: working together for health. Geneva: World Health Organization; 2006.

40. International Council of Nurses/International Hospital Federation/International Pharmaceutical Federation/World Confederation for Physical Therapy/World Dental Federation/World Medical Association. Guidelines: incentives for health professionals. Commissioned by the Global Health Workforce Alliance. Geneva: World Health Organization; 2008.

41. Pierantoli CR, Varella TC, França T. Recursos humanos e gestão do trabalho em saúde: da teoria para a prática. In: Ministério da Saúde, organizador. Observatório de recursos humanos em saúde no Brasil. v. 2. Brasília: Ministério da Saúde; 2004. p. 51-70.

42. Chiavenatto I. Gestão de pessoas: o novo papel dos recursos humanos nas organizações. Rio de Janeiro: Editora Campus; 1999.

43. Cecílio LC, Rezende MFB, Magalhães MG, Pinto SA. O pagamento de incentivo financeiro para os funcionários como parte da política de qualificação da assistência de um hospital público, Volta Redonda, Rio de Janeiro, Brasil. Cad Saúde Pública 2002; 18:1655-63.

44. Reis Neto MT, Assis LOM. Principais características do sistema de remuneração variável no choque de gestão em Minas Gerais: o acordo de resultados e o prêmio por produtividade. Gestão \& Regionalidade 2010; 26:75-90.
45. Santos AF, Ferreira JM, Batista FP, Lages NS. Criando um sistema de informação estratégico em saúde - a experiência do projeto de avaliação de desempenho. In: Reis AT, Santos AF, Campos CR, Malta DC, Merhy EE, organizadores. Sistema Único de Saúde em Belo Horizonte: reescrevendo o público. São Paulo: Xamã; 1998. p. 201-17.

46. Costa MA, Mota JAC, Figueiredo RCP. A nova dinâmica institucional do Hospital das Clínicas da UFMG: o projeto de unidades funcionais e a contratualização com o SUS/BH. Rev Méd Minas Gerais 2009 ; 19:269-75.

47. Rother J. A consumer perspective on physician payment reform. Health Aff 2009; 28:w235-7.

48. Magalhães MFL. Recursos humanos e modelo assistencial - um encontro instigante. In: Reis AT, Santos AF, Campos CR, Malta DC, Merhy EE, organizadores. Sistema Único de Saúde em Belo Horizonte: reescrevendo o público. São Paulo: Xamã; 1998. p. 193-200.

49. Organisation de Coopération et de Développement Économiques. Paying for performance: policies for government employees. Paris: OECD Publishing; 2005

50. Vujicic M, Ohiri K, Sparkes S. Working in health financing and managing the public sector health workforce. Washington DC: World Bank; 2009.

51. Vendemiatti M, Siqueira ES, Filardi F, Binotto E, Simioni FJ. Conflito na gestão hospitalar: o papel da liderança. Ciênc Saúde Coletiva 2010: 15 Suppl 1:1301-14.

52. Matus C. Política, planejamento e governo. Brasília: Instituto de Pesquisa Econômica Aplicada; 1993.

53. Teixeira SMF, Ouverney AM. Gestão de redes. A estratégia de regionalização da política de saúde. Rio de Janeiro: Editora FGV; 2007.

54. Sutton M, McLean G. Determinants of primary medical care quality measured under the new UK contract: cross sectional study. BMJ 2006; 332: 389-90.

55. Narvai PC, São Pedro PF. Práticas de saúde pública. In: Rocha AA, Cesar CLG, organizadores. Saúde pública: bases conceituais. São Paulo: Editora Atheneu; 2008. p. 269-95.

56. Tibério AA, Souza EM, Sarti FM. Considerações sobre avaliação de estabelecimentos de saúde sob gestão de OSS: o caso do Hospital Geral do Grajaú, Saúde Soc 2010; 19:557-68.

57. Reis AAC. Entre a intenção e o ato: uma análise da política de contratualização dos hospitais de ensino (2004-2010) [Tese de Doutorado]. São Paulo: Universidade Federal de São Paulo; 2011.

58. Moreira EMM, Costa EA. Avaliação de desempenho da Agência Nacional de Vigilância Sanitária no modelo de contrato de gestão. Ciênc Saúde Coletiva 2010; 15 Suppl 3:3381-91.

59. Marty IK. Primeiras experiências de contratos de gestão em Curitiba: Secretaria Municipal da Saúde In: Giacomini $\mathrm{CH}$, organizador. Gestão para resultados em Curitiba: a experiência de contratualização. Curitiba: Instituto Municipal de Administração Pública; 2009. p. 43-56. 
60. Ducci L. Curitiba firma contrato de gestão com metas para a saúde. Revista Brasileira Saúde da Família 2007; 8:48-57.

61. World Bank. Brazil enhancing performance in Brazil's Health Sector: lessons from innovations in the state of São Paulo and the city of Curitiba. Washington DC: World Bank; 2006.

62. Ditterich RG. A contratualização como ferramenta de gestão e organização da atenção primária à saúde: avanços, dificuldades e desafios [Tese de Doutorado]. Curitiba: Pontifícia Universidade Católica do Paraná; 2011.
63. Silva MVCP, Tavares Júnior F, Mendes EV. Bases conceituais e programáticas do choque de gestão na saúde em Minas Gerais. In: Marques AJS, Mendes EV, Silva JA, Silva MVCP, organizadores. O choque de gestão na saúde em Minas Gerais. Belo Horizonte: Secretaria de Estado de Saúde de Minas Gerais; 2009. p. 31-46.

Recebido em 16/Ago/2011

Versão final reapresentada em 20/Dez/2011

Aprovado em 07/Fev/2012 